\title{
Leader
}

\section{Micronutrients and immune function: some recent developments}

\author{
David I Thurnham
}

\begin{abstract}
Micronutrient deficiencies probably have few direct effects on the functioning of immune cells. The main effect appears to be a reduction in cell mass that may indirectly affect immune cell function, particularly where $T$ helper cell numbers are reduced. Results of many human studies are contradictory. Some of this contradiction may be accounted for by the fact that disease may lower concentrations of micronutrients in plasma that may be misinterpreted as deficiency. Low plasma vitamin A concentrations however appear to impair immune responsiveness and have deleterious effects on membrane integrity and mucosal function. Zinc may have similar effects on gut integrity and appears to be particularly useful in the treatment of acute diarrhoea. Low concentrations of other nutrients such as ascorbate and iron, may not necessarily impair immune function. Low plasma ascorbate may assist the removal of iron from plasma and low iron concentrations appear to increase the cytotoxicity of macrophages.

(F Clin Pathol 1997;50:887-891)
\end{abstract}

Keywords: micronutrients; immunity; vitamin A; vitamin C; iron; zinc

Since the major reviews of the earlier literature $^{1-3}$ there has been a widespread acceptance in the nutrition community that malnutrition, in the form of protein energy malnutrition or micronutrient deficiencies, impairs immune function and increases the severity of disease. It was therefore somewhat surprising that while browsing through a standard immunology textbook ${ }^{4}$ I could find only one reference to a specific micronutrient. Furthermore, the reference was to the interference in vitamin $B_{12}$ absorption that occurs in pernicious anaemia. Autoantibodies are formed that bind to the patient's intrinsic factor and so prevent vitamin $B_{12}$ from being absorbed. Surprisingly, there was no mention of the other micronutrients such as iron, vitamin $\mathrm{A}$ or $\mathrm{C}$, which have a longer pedigree of involvement in immune function. As malnutri- tion is certainly known to occur in hospitals, ${ }^{5}$ in the elderly, in cancer, and surgical patients, as well as in millions of people living in the developing world where disease and poor health are common, the absence of micronutrients from the immunology text suggests an apparent lack of relevance to the basic components and functioning of the immune system that needs closer scrutiny.

Three fairly large studies illustrate some of the difficulties in interpreting the role of nutritional factors in immunocompetence. Chandra ${ }^{6}$ supplemented 96 apparently healthy and independently living elderly persons (mean age 74 years) with a mixed preparation of physiological amounts of micronutrients in a double blind study over one year. He reported that subjects in the supplemented group had a higher number of certain $\mathrm{T}$ cell subsets and natural killer cells, enhanced proliferation response to mitogen, increased interleukin (IL)-2 production, higher antibody response, and less illness from infection. There were very few associations reported between micronutrient status and the immunological responses in spite of a large number of assays. Of interest were positive relations between serum zinc and IL-2 production and natural killer cell activity, serum vitamin $B_{6}$ positively correlated with lymphocyte response to mitogen, and serum $\beta$ carotene correlated with IL-2 production.

In contrast, Chavance et al ${ }^{7}$ recruited 209 elderly persons (mean age 68 years) of a possible 411 to evaluate the relation between vitamin status and immunological parameters. Recruitment strictly excluded all those whose immune or nutritional status might have been affected by either disease, vaccination or drugs, or who had abnormal erythrocyte sedimentation rates or blood counts. There were two main observations: vitamin $B_{6}$ status was positively correlated with $\mathrm{T}$ cell subsets (CD4 and CD5), and vitamin $\mathrm{E}$ status was negatively related with the number of infections in the previous three years. It should be noticed that the lowest rate of infections corresponded to the lowest vitamin $B_{6}$ status.

Finally, a study reported by Tang et $a l^{8}$ examined the relation between micronutrient intake and the risk of progression to AIDS in 281 HIV positive men. The amount of 
nutrients in the diet had no influence on progression to AIDS. However, if dietary supplements were also considered, thiamin and vitamin $\mathrm{C}$ intakes influenced the progression to AIDS, but only in the final model the levels of niacin and vitamin A (intake $<20000 \mathrm{IU} /$ day) were associated with significantly slower progression to AIDS. Notably, zinc intake was associated with a significantly increased risk of progression.

The studies are different but nevertheless comparisons can be made. In the study reported by Chandra ${ }^{6}$ only $\beta$ carotene and vitamin $E$ were supplemented outside the range of normal dietary intakes. Of these, only $\beta$ carotene was reported as having any link with immune function, but the beneficial effect of zinc status ${ }^{6}$ contrasted strongly with the apparent detrimental effect of zinc on progression to AIDS. ${ }^{8}$ Vitamin $E$ status had no reported impact on immune function in the Canadian study $^{6}$ while in a similar cohort of elderly French people, ${ }^{7}$ it was negatively related to past infections. Vitamin $\mathbf{B}_{6}$ status was the only nutrient that showed some similarities in the two elderly cohorts. It was correlated both with $T$ cell subsets in the French study, ${ }^{7}$ and mitogen stimulation of lymphocytes in the Canadian study, ${ }^{6}$ but had no apparent impact on progression to AIDS. ${ }^{8}$

Quite clearly, large supplements of nutrients may behave in a very different way from those obtained through dietary intake. However, the lack of similarities in the results obtained from elderly French and Canadian populations are a cause for concern. The French group may have been slightly healthier than the Canadians. In the latter, it was reported that immunological responses were greater in those who showed evidence of nutrient deficiency at baseline, which was corrected after 12 months of supplement use. It is interesting to ponder whether the apparent improvements in nutritional status were really a response to the nutrient supplement or represented a response to a change in health. Of the seven "nutrients" where more than $10 \%$ of the population were below the $95 \%$ confidence interval at baseline, five (retinol, vitamin C, iron, zinc, and albumin) are known to be depressed by infections. ${ }^{9}$ Is the contradictory nature of the results a consequence of nutritional status being more influenced by infection and immunological competence rather than the reverse? ${ }^{10}$

\section{Experimental versus human studies}

Poor nutrition and disease have been constant companions of mankind during evolution, and the absolute dependency of an immune response on micronutrient availability would have been evolutionary suicide. The links between micronutrient deficiency and immune impairment are therefore likely to be secondary in character and this is certainly confirmed by results from animal studies. Thus deficiencies of pyridoxine and folic acid (particularly important in protein and nucleic acid metabolism) and vitamins $C$ and $A$, appear to have a wider impact on the immune system (table 1) than most other nutrients. ${ }^{3}$ These nutrients have important effects on integrity and metabolism of many, if not all, rapidly dividing tissues. In contrast, deficiencies of some of the other $\mathbf{B}$ vitamins (riboflavin, thiamin, biotin, and cobalamin) have more specific functions in metabolism and fewer effects on immune function. $^{3}$

However, animal studies on micronutrient deficiencies and immune function differ from many similar studies in man, as in animal experiments the degree and duration of deficiency and/or exposure to infection are known. In contrast, in human studies, duration of or exposure to disease and poor nutrition is rarely known. The assessment of nutritional status is dependent on biochemical markersfor example, plasma or leucocyte ascorbate concentrations, plasma retinol, zinc or iron levels. However, the use of such markers may overestimate the degree of nutritional deficiency, particularly if there is accompanying disease, as disease can depress nutrient concentrations as part of the changes accompanying the acute phase response. ${ }^{9}$ Alternatively, where there is tissue breakdown, plasma concentrations of some B vitamins such as riboflavin and pyridoxine may actually be increased. ${ }^{9}$

\section{Low plasma ascorbate and vitamin $C$ status}

Vitamin C is a good example of a nutrient where experimental deficiencies have shown a consistently increased susceptibility to

Table 1 Response of some immune functions to experimental vitamin deficiencies

\begin{tabular}{|c|c|c|c|c|}
\hline Marker of immune function & Pyridoxine & Folate & Vitamin $C$ & Vitamin $A$ \\
\hline Lymphoid tissue & Generally depressed & & Unchanged & Often atrophic \\
\hline Lymphocyte counts & May be depressed & May be depressed & $\begin{array}{l}\mathrm{T} \text { cell percentages may } \\
\text { decrease }\end{array}$ & May be decreased \\
\hline Antibody production after immunisation & $\begin{array}{l}\text { Consistently depressed primary } \\
\text { and secondary responses }\end{array}$ & May be depressed & Unchanged & May be suppressed \\
\hline $\begin{array}{l}\text { Splenic plaque forming cell response to } \\
\text { immunisation }\end{array}$ & Depressed & May be depressed & & May be suppressed \\
\hline In vitro lymphocyte responses & Diminished in mixed cultures & May be depressed & & $\begin{array}{l}\text { Response to mitogens may } \\
\text { decrease }\end{array}$ \\
\hline Delayed dermal hypersensitivity & $\begin{array}{l}\text { Depressed although sensitisation } \\
\text { mechanism may be intact }\end{array}$ & May be depressed & $\begin{array}{l}\text { Recall mechanism } \\
\text { suppressed but normal } \\
\text { sensitisation remains }\end{array}$ & May be suppressed \\
\hline Allograft survival & Prolonged & & Prolonged & \\
\hline Neutrophil functions & $\begin{array}{l}\text { Inflammatory response may be } \\
\text { diminished }\end{array}$ & May be depressed & $\begin{array}{l}\text { Chemotaxis impaired, } \\
\text { metabolic activity may be } \\
\text { reduced }\end{array}$ & \\
\hline
\end{tabular}


infection, yet the evidence in human studies of disadvantage from deficiency or benefit from supplementation is at best contradictory. Poor vitamin $C$ status is certainly associated with disease but it is difficult to exclude the possibility that disease has interfered with the assessment of vitamin C status. ${ }^{11}$ Vitamin C status is assessed either by measuring the concentration of ascorbate in plasma or blood leucocytes; the latter is considered the better measure of tissue reserves. With the onset of infection, however, there is a rapid increase in the number of blood leucocytes, of which more than $70 \%$ are neutrophils and these enter the circulation from the bone marrow containing very little vitamin $C$. The effect of this sudden increase in leucocytes is a rapid reduction in the blood leucocyte ascorbate concentration as seen following surgery or infection. In one study, ${ }^{12}$ blood leucocyte ascorbate fell more than $50 \%$ following infection with the common cold, irrespective of the level of prior supplementation with vitamin $C$ at the time of infection. (One of the subjects was actually receiving $3 \mathrm{~g}$ vitamin $\mathrm{C}$ each day.) However, in vitro studies have shown that neutrophil granulocytes will rapidly take up vitamin $\mathrm{C}$ from the surrounding medium in a process stimulated by the concentration of vitamin $\mathrm{C}$, antigenic stimulation, and temperature. ${ }^{13}$ Leucocytosis is a normal response to trauma and blood leucocyte ascorbate concentrations usually rectify in three to five days. One of the consequences of disease and the uptake of vitamin $C$ by leucocytes may be the frequent observation of low plasma ascorbate in a wide variety of acute and chronic disease states.

Factors influencing vitamin $\mathrm{C}$ may also influence plasma iron. Trauma and disease have been associated with the presence of free iron in plasma, ${ }^{14}{ }^{15}$ and the lowering of plasma vitamin $\mathrm{C}$ concentrations by the incoming neutrophils may be a desirable process in disease, and one that should not be "corrected" by vitamin $\mathrm{C}$ supplements. For example, ascorbate will react with $\mathrm{Fe}(\mathrm{III})$ reducing it to $\mathrm{Fe}(\mathrm{II})$. Ferrous iron is an undesirable prooxidant and likely to increase tissue damage. ${ }^{14}{ }^{16}$ However, even if the risk of ferrous iron in the plasma in disease is low, the presence of ascorbate is also counterproductive to iron removal. During disease, iron is actively taken up by the reticuloendothelial system ${ }^{17}{ }^{18}$ and an increase in the positive acute phase protein (APP) caeruloplasmin promotes the formation of $\mathrm{Fe}$ (III), the form of iron taken up by ferritin and transferrin. Hence high plasma ascorbate would antagonise the function of caeruloplasmin and prevent iron uptake. ${ }^{14}$

Alternatively, the uptake of ascorbate by neutrophils may increase their cytotoxicity. ${ }^{14}$ There is little evidence available to show that vitamin deficiencies directly influence cytokine production. However, tissue damage may be exacerbated by deficiencies of antioxidant nutrients such as vitamin $E$, vitamin $C$, and $\beta$ carotene. Thus chemotaxis and monocyte activation may be increased by deficiency. To test this hypothesis, Jeng and colleagues ${ }^{19}$ recently studied cytokine production by peripheral blood monocytes (PBMC) following daily supplements of vitamins E (400 mg) and C (1 g). The supplements either singly or in combination were administered for 28 days to healthy volunteers and PBMCs were harvested at intervals to measure peroxides, IL- $1 \beta$, IL- 6 , and tumour necrosis factor (TNF) $\alpha$. The authors reported that only the combined supplement had any effects. IL- $1 \beta$ and TNF $\alpha$ were increased in cultured PMBCs, there was reduced prostaglandin $\mathrm{E}_{2}$ production and increased TNF $\alpha$ following lipopolysaccharide (LPS) stimulation. However, whether these alterations in cytokine production will have any pathophysiological effects in vivo in the diseased state is unknown.

\section{Acute phase response}

Damage to tissues whether from disease or trauma will be indicated by an increase in the positive APP. ${ }^{20}$ Following acute injury there is a delay of about eight hours before the increase in APP. During this period the products of cell damage are detected by monocytes and polymorphonuclear leucocytes, which are stimulated to release cytokines, notably IL-1, IL-2, IL-6, and TNF. It is believed that IL-6 regulates the main APP in the liver but IL-1 and TNF are also involved. ${ }^{21}$

In contrast to the delayed increase in the positive APP, plasma concentrations of the negative APP namely albumin, transferrin, retinol binding protein, and transthyretin, fall almost immediately following trauma. ${ }^{20}$ This rapid response is unlikely to be due to changes in protein synthesis and it is suggested that the products of cell and tissue damage are detected by mast cells. Mast cells will release histamine and increase vascular permeability, and relatively small changes in the distribution of the negative APP between the plasma and extracellular space can account for the large observed changes in these plasma proteins. Subsequently, cytokines reinforce some of the changes by altering hepatic protein synthesis. ${ }^{21}$

Thus low plasma iron and retinol concentrations are initially the consequence of the effects of infection on negative APP. Subsequently, plasma iron remains depressed due to increased uptake by transferrin receptors on reticuloendothelial cells and ferritin in the liver. These effects are consequences of cytokines produced by $T$ cells and macrophages. However, the relative contributions of infection and malnutrition to low plasma concentrations of these nutrients is difficult to evaluate. ${ }^{322}$

\section{Iron}

The evidence from the literature on iron and immunocompetence is far from clear, ${ }^{22}$ and even assessing iron status can be difficult as iron and its binding proteins, transferrin, lactoferrin, and ferritin, are all influenced in one way or another by infection.

Plasma iron falls rapidly with infection, and human studies have shown that this decrease can precede by several days the fever or appearance of clinical signs. ${ }^{17}$ As discussed above the initial fall in transferrin is probably due to the effects of damaged tissue on mast 
cells and the effects of histamine increasing endothelial permeability in the vasculature. ${ }^{20}$ Subsequently cytokines generated by activated $T$ cells and macrophages orchestrate the removal of iron from the plasma and, if infection or trauma continues, are probably responsible for the anaemia of chronic disease. ${ }^{18}$ Monocytes-macrophages are central to this iron removal. Activated T helper cells generate interferon (IFN) $\gamma$ and IL-2, which enhance the expression of transferrin receptors. Furthermore, TNF $\alpha$ and IL-1 induce ferritin synthesis in macrophages and iron is taken up from plasma. ${ }^{18}$ In a similar manner, IL-6 stimulates ferritin synthesis in hepatocytes. ${ }^{18}{ }^{21}$

Low plasma iron therefore is a product of infection and is maintained by the infection, so it is not a surprise that low iron concentrations should be important for macrophage cytotoxicity. ${ }^{18}$ In vitro studies show that low iron stimulates the effects of IFN $\gamma$ on T helper cells and of TNF $\alpha$ in macrophages: the latter by increasing the expression of TNF receptors. The net effect is an increase in macrophage induced cytotoxic effects, part of which may be due to nitric oxide (NO) production. NO has attracted attention in recent years following the demonstration that it is a central component of macrophage mediated cytotoxicity. ${ }^{23}$ In the low iron environment, IFN $\gamma$, TNF $\alpha$, IL- 1 or LPS induce macrophage nitric oxide synthase (NOS). Many of the cytotoxic effects of NO are due to it binding to iron-sulphur clusters in enzymes involved in DNA synthesis and respiration. Obviously the effects of excess iron in such circumstances will both block the production of NO as well as dilute its effect on target cells. Such effects may well explain some of the adverse effects of iron supplements on health in developing countries ${ }^{24}$ and the desirability of using iron chelating drugs in cancer therapy. ${ }^{15}$

\section{Vitamin A: the anti-infection vitamin}

Like vitamin $\mathrm{C}$, deficiencies of vitamin A increase susceptibility to disease, and animal studies have shown that vitamin A deficiency impairs both humoral and cell mediated immunity. ${ }^{3}$ Furthermore, a meta-analysis of a large number of randomised controlled vitamin A supplementation trials concluded that vitamin A has a major role in preventing morbidity and mortality in developing countries. ${ }^{25}$ However, some of the earlier studies on patients with vitamin A deficiency had difficulty in demonstrating impaired immunity. Some of this problem may have been due to patients being inappropriately classed as vitamin A deficient because of depressed retinol levels brought about by disease. For example, Bangladeshi children given 200000 IU vitamin A showed no difference in their antibody response to tetanus toxoid compared with untreated children, ${ }^{26}$ while other studies suggested that vitamin A deficient Indian children had normal cell mediated immunity although the total number of lymphocytes was reduced ${ }^{27}$ It is highly likely in both these communities that a high level of both acute and chronic infection was present, potentially depressing plasma retinol and leading to a false assessment of vitamin status.

Other workers have reported evidence that vitamin A deficiency depresses both humoral immunity such as in measles ${ }^{28}$ and in response to tetanus toxoid treatment, ${ }^{29}$ and cell mediated immunity ${ }^{30}$; the mechanism for these effects is now becoming clear. The main observations in the studies of Semba et al were that children with xerophthalmia (Bitot spots or night blindness) had lower ratios of helper to suppressor lymphocytes and lower proportions of the naive subset of helper $T$ cells than children with normal vitamin A concentrations. ${ }^{30} \mathrm{~T}$ helper cells are important in presenting antigen to $\mathrm{B}$ cells and promoting humoral immunity. Atrophic lymphoid tissue and depressed $T$ lymphocyte numbers are a feature of several micronutrient deficiencies (table 1) and of severe malnutrition in man. ${ }^{10}$ The importance of immunocompetence however is that the functional response to stimulation of the lymphocyte is not impaired. Recent work by Ross ${ }^{31}$ supports the view that immunoglobulin production or cytotoxicity by $B$ cells and natural killer cells, respectively, is not impaired in vitamin A deficient animals in spite of depressed $\mathrm{T}$ lymphocyte numbers, only the magnitude of the response is depressed. Thus it would appear that the principal effects of vitamin $A$ inadequacy on immune function may be a consequence of impaired growth and differentiation of myeloid tissues.

Is vitamin A deficiency more important than other nutrients necessary for cell replication such as vitamin $B_{6}$ or folic acid? In poor communities the dietary source of all three nutrients are vegetable products yet neither $B_{6}$ nor folate deficiencies have been linked with immunodeficiency in man to any great extent. In contrast vitamin $\mathrm{A}$ has been labelled the anti-infection vitamin from early this century and the reason for this may be due to the depression in plasma retinol caused by infection. The depression in serum retinol may expose an individual to inadequate plasma vitamin A concentrations in areas where the dietary intake was already marginal. In particular, vitamin $\mathrm{A}$ is specifically important for the integrity of epithelium and the maintenance of mucosal secretions which, if impaired, may increase exposure to microorganisms and increase the risk of infection.

\section{Vitamin $A$ and tissue integrity}

Experimental studies have shown that epithelial tissues of the eyes, lungs, and gut are impaired by vitamin A deficiency. These are all tissues where epithelial cell turnover is high. In man, numerous studies using the impression cytology test have shown that low circulating vitamin $A$ is associated with an increased risk of epithelial damage in the eye. ${ }^{9}$ Impaired gut integrity is common in malnutrition ${ }^{10}$ and was shown to explain more than $40 \%$ of growth faltering in Gambian infants ${ }^{32}$ but was at its best in the Gambian study, during the mango season. ${ }^{33}$ Mangoes are an important sources of vitamin $A$, and we have recently obtained evidence that weekly vitamin A supplements 
(equivalent to 1.5 times the recommended daily allowance) significantly improved gut integrity in 40 Indian infants over eight weeks (McCullough et al, unpublished data, 1997). The study was done during the rainy season when diarrhoea, respiratory disease, malaria, and other diseases are often at their highest. Damage to the integrity of epithelia and mucosal barriers will facilitate translocation of microorganisms and contribute to the increased severity of infections. Thus low plasma vitamin A may compromise immune function by impairing epithelial integrity and by depressing lymphocyte numbers, and although the capacity of immune cells may still be normal, ${ }^{31}$ the overall immune response is depressed.

\section{Zinc}

Zinc is another nutrient for which status is difficult to evaluate. Zinc serum concentrations, like vitamin $\mathrm{A}$ and iron, are depressed by infection and trauma. Infusions of IL-1 into animals resulted in decreases in plasma iron and zinc, and incubating isolated hepatocytes with IL-1 increased transcription of metallothionen, ${ }^{21}$ an important zinc binding protein. However, only about $1 \%$ of total body zinc is found in the serum, ${ }^{34}$ so large changes in the blood may mean very little in overall body zinc status.

Zinc does however show other similarities with vitamin A. Grossly abnormal epithelial structure and function is a feature of the zinc deficiency syndrome acrodermatitis enterohepatica, and early studies showed that zinc supplements resulted in striking reductions in diarrhoea and improvements in mucosal function. ${ }^{35}$ More recent work has confirmed the benefits of zinc supplements in young children with acute diarrhoea ${ }^{36}$ and other studies have demonstrated improvements in gut integrity of malnourished infants using zinc. ${ }^{37}$ Zinc has been shown to stimulate the synthesis of retinol binding protein and it is possible that the effects of zinc may be via improvements in vitamin A status.

\section{Conclusions}

Experimental studies clearly show that many specific micronutrient deficiencies depress myeloid tissue growth and impair tests of immunocompetence; however, it is usually not clear whether the effects are due to reduced numbers of immune cells or functional damage to the immune cell. Studies in man to relate nutritional status to changes in immune function have generally produced inconsistent results with the exception of vitamin $A$, where most supplementation studies in malnourished populations have shown benefits in both morbidity and mortality. A complicating factor in interpreting human studies is the assessment of nutritional status as disease can influence many of the indices currently in use. Vitamins $A$ and $C$, and minerals iron and zinc are particularly influenced by disease, complicating interpretations of the role these micronutrients may play in immune function.
1 Chandra RK, Newberne PM. Nutrition, immunity and infection. Mechanisms of interactions. New York: Plenum Press, 1977.

2 Gross RL, Newberne PM. Role of nutrition in immunologic function. Physiol Rev 1980;60:188-302.

3 Beisel WR. Single nutrients and immunity. Am $\mathcal{F}$ Clin Nutr 1982;35:415-68.

4 Roitt I, Brostoff J, Male D. Immunology. 2nd edn. London: Churchill Livingstone, 1990.

5 McWhirter JP, Pennington CR. Incidence and recognition of malnutrition in hospital. $B M \mathcal{F}$ 1994;308:945-8.

6 Chandra RK. Effect of vitamin and trace-element supplementation on immune responses and infection in elderly subjects. Lancet 1992;340:1124-7.

7 Chavance M, Herbeth B, Fournier C, Janot C, Vernhes G. Vitamin status, immunity and infections in an elderly population. Eur f Clin Nutr 1989;43:827-35.

8 Tang AM, Graham NMH, Kirby AJ, McCall LD, Willet WC, Saah AJ. Dietary micronutrient intake and risk of progression to aquired immunodeficiency syndrome (AIDS) in human immunodeficiency virus type 1 (HIV-1)-infected homosexual men. Am $¥$ Epidemiol 1993;138:937-51.

9 Thurnham DI. Impact of disease on markers of micronutrient status. Proc Nutr Soc 1997;56:421-31.

10 Morgan G. What, if any, is the effect of malnutrition on immunological competence. Lancet 1997;349:1693-5.

11 Thurnham DI. $\beta$-Carotene, are we misreading the signals in risk groups? Some analogies with vitamin C. Proc Nutr Soc 1994;53:557-69.

12 Hume $R$, Weyers $E$. Changes in leucocyte ascorbic acid during the common cold. Scot Med f 1973;18:3-7.

13 Moser U, Weber F. Uptake of ascorbic acid by human granulocytes. Int $\mathcal{F}$ Vit Nutr Res 1984;54:47-53.

14 Thurnham DI. Antioxidants and prooxidants in malnourished populations. Proc Nutr Soc 1990;48:247-59.

15 Beare S, Steward WP. Plasma free iron and chemotherapy toxicity. Lancet 1996;347:342-3.

16 Halliwell B, Gutteridge JMC. Biologically relevant metal ion-dependent hydroxyl radical generation. An update. FEBS Lett 1992;307:108-12.

17 Beisel WR. Trace elements in infectious processes. Med Clin North Am 1976;60:831-49.

18 Weiss G, Wachter H, Fuchs D. Linkage of cell-mediated immunity to iron metabolism. Immunol Today 1995;16: 495-500.

19 Jeng K-CG, Yang C-S, Siu W-Y, Tsai Y-S, Liao W-J, Kuo J-S. Supplementation with vitamins $C$ and $E$ enhances J-S. Supplementation with vitamins $C$ and $E$ enhances cytokine production by peripheral blood mononuc

20 Fleck A. Clinical and nutritional aspects of changes in acute-phase proteins during inflammation. Proc Nutr Soc 1989;48:347-54

21 Warren JS. Interleukins and tumor necrosis factor in inflammation. Clin Rev Clin Lab Sci 1990;28:37-59.

22 Chandra RK. Iron and immunocompetance. Nutr Rev 1976;34:129-32.

23 Moncada S, Palmer RMJ, Higgs EA. Nitric oxide physiology, pathophysiology and pharmacology. Pharmacol Rev 1991;43:109-42.

24 Oppenheimer SJ. Iron and malaria. Parasitology Today 1989; 5:77-9.

25 Glasziou PP, Mackerras DEM. Vitamin A supplementation in infectious diseases: a meta-analysis. BMF 1993;306:36670.

26 Brown KH, Muang Rajan M, Chakraborty J, Aziz KMA Failure of a large dose of vitamin A to enhance the antibody response to tetanus toxoid in children. Am $\mathcal{F}$ Clin Nutr 1980;33:212-17.

27 Bhaskaram C, Reddy V. Cell-mediated immunity in iron and vitamin deficient children. $B M 71975$;iii:522.

28 Coutsoudis A Kiepiela P, Coovadia HM, Broughton M. Vitsoudin A , Kiepiela $P$ Coon bers while improvin morbidity in measles. Pediatr Infect Dis f 1992;11:203-9.

29 Semba RD, Muhilal, Scott AL, Natadisastra G, Wirasmita $S$, Mele L, et al. Depressed immune response to tetanus in children with vitamin A deficiency. $\mathcal{F}$ Nutr 1992;122:101-7.

30 Semba RD, Muhilal, Ward BJ, Griffin DE, Scott AL, Natadisastra $\mathrm{G}$, et al. Abnormal T-cell subset proportions in vitamin A deficient children. Lancet 1993;341:5-8.

31 Ross AC. Vitamin A deficiency and retinoid repletion regulate the antibody response to bacterial antigens and the maintenance of natural killer cells. Clin Immunol Immunopathol 1996;80:S63-72.

32 Lunn PG, Northrop-Clewes CA, Downes RM. Intestinal permeability, mucosal injury and growth faltering in Gampermeability, mucosal injury and grow

33 Northrop-Clewes CA, Lunn PG, Downes RM. Seasonal fluctuations in vitamin A status and health indicators in Gambian infants [abstract]. Proc Nutr Soc 1994;53:144A.

34 Aggett PJ, Favier A. Zinc. Int $\mathcal{F}$ Vit Nutr Res 1993;63:301-7.

35 Tomkins A, Behrens R, Roy S. The role of zinc and vitamin $A$ deficiency in diarrhoeal syndromes in developing countries. Proc Nutr Soc 1993;52:131-42.

36 Sazawal S, Black RE, Bhan MK, Bhandari N, Sinha A, Jalla $S$. Zinc supplementation in young children with acute diarrhea in India. $N$ Engl $f$ Med 1995;333:839-44.

37 Roy SK, Behrens RH, Haider R, Tomkins AM. Impact of zinc supplementation on intestinal permeability in Bangladeshi children with acute diarrhoea and persistent diardeshi children with acute diarrhoea and persistent diar96. 